\title{
Erwiderung auf den vorstehenden Artikel des Herrn Landmann.
}

Von

\author{
Prof. Dr. H. Sahli in Bern.
}

Der vorstehende Aufsatz des Herrn Land ma $n$ n enthält eine Menge ron Missverständnissen und Entstellungen dessen, was ich in meiner Arbeit über Tuberkulinbehandlung gesagt habe und ich verwoise diejenigen, welche meine Ansichten unkarrikiert kemmen zu lernen wünschen, auf meine Originalmitteilung ${ }^{\mathbf{1}}$ ). Ich habe von den dort geäusserten Ansichten nichts zurückzunehmen. Fine eingehende polemische Diskussion mit Herrn Lan $\mathrm{d}_{\mathrm{m}}$ an $n$ ist namentlich schon deshalb wenig erspriesslich, weil derselbe die zum t'eil neuen (irundgedanken meiner Arbeit nicht verstanden hat und wohl auch nicht verstehen will. Da aber zu fürchten ist, dass seine entstellenden Auseinandersetzungen Verwirrung stiften, so halte ich im Interesse der guten Sache, der ich zu dienen glaube, mich doch zu einer etwas eingehenderen Erwiderung verpflichtet, so wenig auch eine solche Iolemik meinen Neigungen entspricht.

Ich resümiere hier zunächst nochmals die wichtigsten Grunclgedanken meiner Arbeit. Es sind folgende: Die Tuberkulinbehandlung soll eine funktionelle Beeinflussung des Körpers bewirken, vermöge deren letzterer die toxischen Komponente des Krankheitsvorganges durch die Mehrbildung von Antikörpern leichter überwindet. Mit einer solchen immunisatorischen Entgiftung ist aber noch keineswegs eine Heilung der Tuberkulose notwendig verbunden, demn die toxischen Vorgänge spielen bei der Tuberkulose keineswegs die

1) Über Taberkulinbehandlung. Basel, B. Schwalbe, Verlagsbuchhandlung. 1907. 2. Auflage. 
hervorragende und alleinherrschende Rolle wie etwa bei der Diphtherie. Der Nachweis, dass trotz hoher Gift- oder Tuberkulinfestigung eine Tuberkulose sich verschlimmem kann und dass umgekehrt eine Tuberkulose ohne jede Tuberkulinfestigkeit, d. h. bei hoher Tuberkulinempfindlichkeit heilen kann, ist klinisch tausendfach erbracht und von fundamentaler Bedeutung für die ganze Frage. Antitoxische Immunisierungsvorgänge spielen deshalb bei der Heilung dieser Krankheit zwar eine Rolle, aber nicht die nämliche herrschende wie bei der Diphtherie. In völliger Übereinstimmung damit ist für die Möglichkeit einer vollkommenen aktiven Immunisierung gegen lebendige 'Tuberkelbazillen durch chemische Mittel weder klinisch noch experimentell bisher ein Beweis geliefert worden. Im Gegenteil sprechen die Erfahrungen bei der 'Tuberkulinbehandlung dafür, dass dieselbe bloss eine zuweilen allerdings entscheidende Hilfe für die Naturheilung darstellt. Iyie Tuberkulinbehandlung ist demnach für den einzelnen Fall indiriduell und rationell zu dosieren und hat keineswegs dem bisher unerreichbaren Ziel einer absoluten Immunisierung nachzustreben. Aufgabe der Tuberkulinbehandlang ist es, in den geeigneten Fällen, die ich seinerzeit präzisiert habe, das zu der betreffenden Zeit in Körper vorhandene chemische Tuberkulosegift. durch gesteigerte Produktion rom Antikörpern unsehädlich zu machen und dadurch die hauptsächlich durch den Fitzündungsprozess vermittelte Naturheilung zu erleichtern. Wenn wir über die hierzu erforderliche, oft erstaunlich niedrige Dosierung des Tuberkulins durch progressive Dosensteigerung prinzipiell im allgemeinen hinausgehen, so hat dies bloss den Zweck, den Organismus auch gegen eventuelle spätere Krankheitsschübe zu schützen, etwa ähnlich wie ein kluger Haushalter bei seinen Einkäufen auch an die Zukunft denkt. Ausserdem wissen wir ja in den Fällen, welche unter der Tuberkulinbehandlung nicht rasch eine günstige Wendung nehmen, nicht immer, wann die Antikörperproduktion eine genügende geworden ist und wir suchen sie deshalb im allgemeinen möglichst hoch zu treiben, um sicher zu sein, dass wir unseren Zweck, die Giftkomponente unschädlich zu machen, erreichen. Wegen der mit den höheren Tuberkulindoser oft verbundenen Gefahr der Giftüberlastung ist es aber zweckmässig, nicht einem Prinzipe zuliebe zu weit zu gehen, sondern da, wo sich für die Steigerung der Dosen Schwierigkeiten ergeben, sich mit dem für den gegebenen Moment erreichbaren individuellen Maximum oder Optimum zu hegnïgen, nach dem alten Crrundsatz: Das Bessere ist oft der Feind des Guten. Als individuelles Ma $\mathrm{x}$ i m $\mathrm{u}$ m habe ich die maximale Dosis Tuberkulin bezeichnet, welche der Kranke ohme reaktive Erscheinungen erträgt, als individuelles Opti- 
m u m die häufig damit übereinstimmende, zuweilen aber auch etwas niedrigere Dosis, bei welcher er die besten therapeutischen Fortschritte macht. Ich habe für die geschilderte therapeutische Einwirkung des Tuberkulins den Begriff der immunisatoris chen $\mathrm{Heilwirkung}$ eingeführt. Dieselbe ist toto coelo verschieden von demjenigen, was man bei der Immunisierung eines Serum spendenden Tieres zu erzielen sucht, mit welcher Herr La $n d m$ a $n n$ in völliger Verkennung der Sachlage immer argumentiert. Da dieser Begriff der immunisatorischen Heilwirkung vielleicht, weil er neu ist, nicht überall und jedenfalls nicht bei Hernn Landmann das nötige Verständnis finden wird, so möchte ich hier darauf hinweisen, dass ich diesen Begriff nicht konstruiert, sondern ihn gewissermassen der Natur abgelauscht habe. Immunisatorische Heilwirkungen im Sinne meiner Auffassung sind nämlich überall da bei der natürlichen Heilung von Infektionskrankheiten im Spiele, wo eine Krankheit, wie z. B. die Pneumonie oder das Erysipel, offenbar durch Immunisationsvorgänge, wofür schon die kritischen Erscheinungen sprechen, heilt, ohne dass dies zur Immunität führt. Diese paradoxe Tatsache Heilung einer Infektion ohme Eintreten von Immunität - erklärt sich am einfachsten durch die Annahme, dass die Antikörperproduktion bei diesen Krankheiten zwar genügt, um das Gift momentan unschädlich zu machen und dadurch die heilende Wirkung zu entfalten, respektive den übrigen Heilfaktoren, der Entzündung, den Leukozyten usw. die Heilung zu ermöglichen, dass aber dabei keineswegs immer ein dauernd erhöhter Antikörperbestand oder auch nur die Fähigkeit des Organismus, in Zukunft neue Infektionen durch sofortiges Mobilmachen von Antikörpern zurückzuweisen, zurückbleibt. Diese Vorkommnisse finden sowohl in dem günstigen natürlichen Verlauf der Tuberkulose, als auch in dem durch Tuberkulinbehandlung günstig modifizierten Verlauf derselben ihre Analoga. Um eigentliche Immunität handelt es sich dabei nicht. Ich hoffe, damit die prinzipielle Differenz von Immunisation und immunisatorischer Heilwirkung klar gemacht zu haben. Ohne die Berücksichtigung dieses Unterschiedes entbehrt die Tuberkulinbehandlung einer rationellen Basis und wird stets wieder durch Giftüberlastung zu Unheil führen. Ich bemerke noch, dass dasjenige, was ich als immunisatorische Heilwirkung bezeichne, manchem Leser vielleicht durch die Bezeichnung Stimulation oder Anregung der natürlichen Heilvorgänge durch Mobilisierung von Antikörpern verständlicher werden dürfte, und ich gestehe, dass der von mir der Immunisationslehre entnommene und in die Lehre von der Tuberkulinbehandlung eingeführte Ausdruck der Giftfestigung, obschon er, wenn man ihn so versteht 
wie ich, richtig ist, doch gerade wegen der Möglichkeit einer Verwechslung zwischen Immunisation und immunisatorischer Heilwirkung zu Missverständnissen Anlass geben kann.

Als Methode der Dosensteigerung für diese immunisatorische Heilwirkung empfahl ich ein ganz allmähliches mildes, alle klinisch manifesten sogenannten Reaktionen vermeidendes Verfahren, wie es sich am leichtesten mittelst der für das Berane cksche Tuberkulin adoptierten Verdünnungsskala durchführen lässt, die natürlich sich auch für andere Tuberkuline eignet. Das auch mir sehr wohl bekannte Gesetz, dass zur Erzeugung kräftiger immunisatorischer Wirkungen schubweise plötzliche Einwirkungen des Antigens gegenüber der gleichmässigen Giftwirkung, wie sie die Krankheit an sich bedingt, erforderlich sind, wird durch dieses milde Verfahren keineswegs verletzt. Denn mit jeder Injektionsbehandlung sind plötzliche Einwirkungen notwendigerweise verbunden, auch wenn sie grob-klinisch nicht zu erkennen sind. Wir können sie, wie so vieles in der Medizin, nur mit dem Auge des Geistes, darum aber nicht minder sicher sehen. Die schubweisen heilsamen Reaktionen fehlen also bei einer solchen milden Tuberkulinbehandlung keineswegs, sie spielen sich bloss jenseits der Schwelle der klinischen Wahrnehm. barkeit ab. Und sollte Herr Landmann über das ,Auge des Geistes" wie über so vieles andere den Kopf schütteln, so nehme ich es ihm nicht übel. Den Lesere möchte ich dagegen darauf hinweisen, dass auch bei der Heilung anderer Infektionskrankheiten solche Reaktionen, die nur das geistige Auge erkennt, nach allgemein akzeptierter Auffassung eine entscheidende Rolle spielen. Wer hat je in dem klinischen Bilde eines Typhus grobklinisch die immunisatorischen Reaktionen ges ehen, welche sicherlich auch bei dieser Krankheit die Heilung hervorrufen? Die durch Traube nachgewiesenen kritischen Tage der Pneumonie ferner weisen mit Bestimmtheit auf immunisatorische Reaktion en hin und zwar auf solche, die sich in zweitägigen Perioden wiederholen. Und doch merkt man von diesen Reaktionen für gewöhnlich nichts und nur in Ausnahmefällen macht sich die letzte für die Heilung entscheidende Reaktion in Form einer „Perturbatio critica" als vorübergehende Verschlimmerung des Zustandes auch klinisch geltend. Auch aus der experimentellen Pathologie lassen sich viele Beispiele anführen, dass reichliche Antikörperproduktion ohne alle manifesten sogenannten Reaktionserscheinungen stattfinden kann. So fand Knorr, dass , bei dem wenig tetanusempfindlichen Huhn schon der zehnte Teil einer (Xiftmenge, die noch keine Krankheitserscheinungen macht, zur lebhaften Produktion von Antikörpern Anlass gibt." Ich bin also der Ansicht, dass jede 
injektorische Tuberkulinbehandlung, auch die mildeste, besonders da sie sich bei der allmählichen Dosensteigerung im allgemeinen an der Grenze der Toleranz bewegt, solche latente reaktive Vorgänge weckt und ich bin auch vollständig damit einverstanden, dass Reaktionen in diesem Sinne nach allem, was wir über das Wesen der Immunisierung wissen, für den Heileffekt wesentlich sind. Etwas ganz anderes aber ist das grobe Geschütz der klinisch manifesten Reaktionen im gewöhnlichen Sinne des Wortes. Vor diesen warne ich! Sie sind der Ausdruck einer weitergehenden Schädigung des Organismus. Sie können zwar vielleicht - das will ich nicht bestreiten günstige Folgen haben, etwa ähnlich wie die Perturbatio critica einer Pneumonie, sie müssen es alyer nicht. Auch bei der Pneumonie kann der Mensch an der als Perturbatio critica bezeichneten Reaktion zugrunde gehen. Die Wirkung solcher klinisch sichtbarer Reaktionen ist im allgemeinen zweischneidig. Sie sind deshalb für den Arzt, der womöglich nur nützen und nicht schaden möchte, soviel als möglich zu vermeiden und haben tatsächlich die Tuberkulinbehandlung immer wieder diskreditiert und werden es auch fernerhin tun.

In betreff der Wahl des Tuberkulins habe ich mich ausdrücklich und im Gegensatz zu der Landmann schen Darstellung dahin ausgesprochen, dass man mit jedem Tuberkulin, wenn man es richtig handhabt, günstige Resultate erhalten kann, da wohl alle Tuberkuline eine gemeinsame Komponente besitzen. Ich habe dabei aber bemerkt, dass mir das Beranecksche Tuberkulin nicht bloss wegen der gebrauchsfertigen Form, sondern auch wegen seiner theoretischen resp. experimentellen Begründung vor allem empfehlenswert erscheine. Was ich unter dieser theoretischen Begründung verstehe, über welche Lan $\mathrm{d}$ man $n$ die ganze Schale seines Zornes ausgiesst, habe ich für den aufmerksamen Leser meiner Arbeit, wenn auch, da dies nicht mein eigentliches Thema war, nur kurz angegeben. Nur Land$m$ a $n n$ will es nicht verstehen. Indem er hervorhebt, dass $B$ e ran e c $k$ auf die phagozytäre Wirkung des Tuberkulins grosses Gewicht lege, während ich vorwiegend von der Erzeugung von Antikörpern durch die Tuberkulinbehandlung spreche, glaubt er merkwürdigerweise einen unvereinbaren Widerspruch zwischen den Ansichten Beranecks und den meinigen festzustellen, ja er meint sonderbarerweise sogar, jeh ,verspotte" Beraneck wegen seiner Ansicht. Damit ist num für Landmann die theoretische Begründung des Tuberkulin Beraneck als widerspruchsvoll und ungenügend abgetan. Fr erwähnt dabei nicht, dass ich in meiner Arbeit ausdrücklich dic Wirkung der Leukozyten, wenn auch in einem viel allgemeineren Sinn 
als dem der blossen Phagozytose, die mir keine genügende Erklärung zu sein scheint ${ }^{1}$ ), durchaus anerkenne, dass ich sie aber, so weit sie durch die Tuberkulinbehandlung beeinflusst wird, wie die gesamten übrigen therapeutischen. Wirkungen des Tuberkulins auf die Anregung der Antikörperproduktion zurückführe. Ich anerkenne ja auch die 'Tatsachen der sogenannten opsonischen Wirkungen, d. h. der Wirkung der Antikörper auf die Phagozytose, wenn ich auch den Ausdruck Opsonine, welcher falsche Vorstellungen erwecken kann, für überflüssig halte und diesen Begriff in demjenigen der Antitoxine aufgehen lasse. Der Unterschied zwischen den Ansichten Berane cks und den meinigen ist also gar nicht gross und betrifft jedenfalls nur Details. Auch erwähnt Landmann nicht, dass Beraneck in sciner zweiten Arbeit ${ }^{2}$ ) die Wirkungen des Tuberkulins ebenfalls wesentlich auf Antikörperproduktion zurückführt, die er denn auch vollständiger als es bisher für andere Tuberkuline geschehen ist, gerade für sein Tuberkulin in der Form von Agglutininen, Ambozeptoren, Präzipitinen und Antitoxinen im Serum eines mit seinem Tuberkulin immunisierten Pferdes nachgewiesen hat. Speziell hat Berane $\mathrm{ck}^{3}$ ) auch gezeigt, dass das Serum der mit seinem Tuberkulin vorbehandelten Pferde sowohl eine die toxische Wirkung seines Thuberkulins herabsetzende, als beim Zusammenbringen mit Tuberkelbazillen eine direlite, die Virulenz der ersteren herabsutzende Wirkung besitzt ${ }^{4}$ ).

So konstruiert Landman $n$ im Interesse seiner Polemik und nach dem alten Grundsatz Divide et impera einen prinzipiellen Gegensatz unserer Ansichten, der gar nicht existiert, nur um sagen zu können, meine Behauptung einer guten theoretischen Begründung des Tuberkulin. Beraneck sei unwahr. Aber kommt es denn über-

1) Meine frïher geäusserte Ansicht, dass die Leukozyten auch noch in anderer Weise als durch Phagozytose für den Ablauf von Infektionskrankheiten eine nützliche Wirkung haben können - wie ich mich ausdrückte, als bindungsfähiges oder affinitätentragendes Eiweissmaterial - ist durch eine neue Untersuchung von M. Gruber und Kenzo Futaki über Resistenz gegen Milzbrand ( $L$ Leuk. anthracocidin" und "Plakanthracocidin" vgl. Deutsche med. Wochenschr. 1907. Nr. 39) nun auch experimentell genügend gestützt.

2) Schweiz. Ärzteversammlung in Neuenburg vom 25. Mai 1907, abgedruckt in der Revue med. de la Suisse Romande 1907. Nr. 6. 20. Juni.

3) Revue de la Suisse Romande, 20. Oct. 1905, Nr. 10.

4) Ferner hat er den Nachweis erbracht, dass sein Tuberkulin als solches die Eigenschaft hat, die Virulenz von mit demselben digerierten Tuberkelbazillen abzuschwächen, und dass diese Eigenschaft nicht etwa bloss auf seinen Orthophosphorsäuregehalt, sondern auf eine spezifisch antagonistische Beeinflussung der 'Tuberkelbazillen durch ihre eignen Stoffwechselprodukte zurückzuführen ist. 
haupt bei dieser Frage der theoretischen Begründung auf Ansichten an und nicht vielmehr auf Tatsachen? Liegt denn nicht, wie ich es ausdrücklich, wenn auch kurz, auf Seite 3 meiner Arbeit erwälnte, die theoretische Begründung des Tuberkulin Beraneck gerade in Tatsachen, nämlich in den experimentellen Resultaten Beranecks? Auf diese muss ich nun noch etwas näher eingehen, da sie Landmann im Interesse seines Angriffes teils entstellt, teils einfach rerschweigt.

Berane $\mathrm{ck}$ hat, abgesehen von dem schon erwähnten Nachweis aller Arten von Antikörpern in dem Serum seiner Tuberkulinpterde, experimentell in erster Linie gezeigt, dass sein Tuberkulin die immunisierenden Bestandteile von T'uberkulosekulturen möglichst vollständigr und rein, d. h. wenig belastet mit schädlichem Ballast nicht spezifischer und dennoch giftiger Substanzen enthält, und zweitens, dass das Präparat überhaupt trotz seiner von $\mathrm{Beraneck}$ an Meerschweinchen nachgewiesenen schützenden Eigenschaften für das gos o n d e Meerschweinchen relativ wenig giftig ist. Von diesen experimentellen Feststellungen erwähnt Landmann bloss einige Fragmente, die er so weit entstellt, dass er sie für seine Polemik benutzen kann. Ich führe deshalb speziell folgendes an:

1. Die Inmunisierungs- und Heilversuche an Meerschweinchen haben für das Tuberkulin Bera n e e k zwar kein absolutes aber doch cin günstigeres Resultat (beträchtlichere Lebensverlängerung) ergeben als die meisten entspreehenden Versuche mit anderen Tuberkulinen, welche in der Literatur mitgeteilt sind, und als die Versuche, welche Beraneck selbst mit anderen Tuberkulinen vorgenommen hat.

2. Die relativ geringe Giftigkeit des Tuberkulin Beraneck ergibt sich daraus, dass die gesunden Meerschweinchen die beträchtliche Menge von $10 \mathrm{ccm}$ des „,konzentrierten“ Tuberkulin B e ra n e ck ertrugen und dass das Präparat auch von tuberkuläsen Meerschweinchen, was ja für die Frage der Behandlung kranker Menschen ron Interesse ist, in relativ grossen Mengen ertragen wurde. Nach mïndlicher Mitteilung, die mir Herr B e r a n e ck machte, tötet crst die Menge von $1 \mathrm{ccm}$ des ,konzentrierten“ Tuberkulin Beraneck das tuberkulöse Meerschweinchen. Ich muss dabei hervorheben, dass dieses ,konzentrierte Tuberkulin Beraneck" nicht im Handel ist und nicht verwechselt werden darf mit dem, was ich in meiner Arbeit als reines Tuberkulin Beraneck (Lösung $\mathrm{H}$ ) bezcichnet laabe, sondern noch $62,4 \mathrm{mal}$ konzentrierter ist als dieses. Wenn man dies barücksichtigt, so sind die erwähnten für das tuberkulöse Meerschweinchen tötlichen Dosen von einer Grössenanordunug, wie sie für die Behandlung des kranken Menschen nach der 
für das Beranecksche Tuberkulin erprobten Technik nicht in Betracht kommt. Ich betone dabei, dass natülich nur diese Art der Bestimmung der Giftigkeit eines Tuberkulins, nämlich der Giftigkeit im Verhältnis zu den für die menschliche Therapie in Betracht kommenden Dosen, einen praktischen Wert hat. Die Vergleichung der Giftigkeit der einzelnen Tuberkuline untereinander nach absoluten Gewichts- oder Volummengen hat dagegen, wie ich schon in meiner ersten Arbeit andeutete, gar keinen Wert, resp. gar keinen Sinn, weil die Tuberkuline je nach der Art ihrer Darstellung nicht bloss qualitativ chemisch, sondern auch an Konzentration sich erheblich unterscheiden. Dabei ist aber selbstverständlich nur von einer relativ geringen Giftigkeit des Beraneckschen Tuberkulins die Rede, wie aus der Angabe B e ra n e cks deutlich hervorgeht, dass das Präparat in der Dose von $1 \mathrm{ccm}$ das tuberkulöse Meerschweinchen töte. Es ist deshalb schwer verzeihlich, dass $L$ a n $d m$ a $n n$ das Tuberkulin Beraneck durch die gänzlich aus der Luft gegriffene Behauptung zu diskreditieren sucht, das Präparat töte tuberkulöse Meerschweinchen überhaupt nicht und sei schon aus diesem Grunde wertlos (sic!). Die Feststellung, dass das Tuberkulin Beraneck für das tuberkulöse Mcerschweincheil ganz ungiftig sei, wäre in der Tat für ein immunisierendes Mittel etwas bedenklich oder, besser gesagt, da die schützende Wirkung durch Beraneck doch nachgewiesen wurde - sie wäre ,allzuschön gewesen". Es handelt sich aber wie gesagt hier um eine reine Erfindung Land hin an $11 \mathrm{~s}$. Weshalb nun Landmann, der doch auf starke Giftigkeit der Tuberkuline so grossen Wert legt, dass er sagt, es sei sein eifrigstes Bestreben, die Giftigkeit seines Tuberkulins noch zu steigern, sich darüber aufregt, dass ich sein Tuberkulin, mich an seine eigenen Angaben haltend, als hervorragend giftig bezeichne, ist schwer verständlich und entbehrt jeder Logik.

3. Von dem Momente an, wo Beraneck für sein Präparat nach seinen Meerschweinchenversuchen ein die meisten anderen Tuberkuline übertreffendes Immunisationsresultat und dabei eine relativ geringe Giftigkeit desselben gefunden hatte, war er offenbar im Rechte, in dem Weglassen des Peptonzusatzes zu seiner Kulturbrühe, das zu diesem Resultate geführt hatte und ein wesentliches Merkmal des Beraneckschen Tuberkulins ist, einen Vorteil zu sehen. Die spitzfindigen Erörterungen La n d $\mathrm{m}$ a $n \mathrm{n}$ s, dass sich beim Sterilisieren der Bouillon dennoch Pepton bilde, dass also das Bera n e cksche Tuberkulin doch nicht ganz peptonfrei sei, was natürlich B e r a n e ck sehr wohl bekannt ist, ändern daran nichts. Es kommt doch eben auch auf die Mengenverhältnisse des Peptons an, wenn von den 
Giftwirkungen dieser Substanz die Rede ist und die Angabe Bera n ecks, dass durch die Weglassung des Peptonzusatzes eine zum mindesten ganz überflüssige Giftkomponente wegfällt, besteht also zweifellos zu Recht.

4. Die Behauptung Landmanns, das Berano cksche Tuberkulin beanspruche die spezifisch toxischen Substanzen der Tuberkulosekulturen vollständig und vollständig unverändert zu enthalten, jst wieder eine der Unrichtigkeiten, welche der I, a n d m a n n sche Artikel enthält. Fs war immer nur die Rede (vol. meine Tuberkulinarbeit, 2. Aufl. S. 3) ron einer $\mathrm{m} \ddot{\mathrm{g}} \mathrm{l}$ i chs t. rollständigen und $\mathrm{m} \ddot{\mathrm{g}} \mathrm{-}$ lichst unveränderten Extraktion und zwar nicht der Gifte äberhaupt, sondern der fuir die Immunisationswirkung in Betracht kommenden Substanzen, von denen, wie ich zeigen werde, durchaus nicht rerlangt zu werden braucht, dass sio besonders stark toxisch sind. Ob ein diesen Forderungen entsprechendes Präparat rorliegt, darüber geben vorläutig die Experimente Boranecks und die kilnische Erfahrung Aufschluss, nicht die Spekulationen La ndmanns.

Dies ist die theoretische Begrïndung des Tuberkulin B er a n e ck, von der ich in meiner Arbeit sprach. Sie wird allerdings Landma $n \mathrm{n}$ auch nach dicsen Frklärungen kaum genügen. Ienn dieser Autor hat über das Wesen einer zweekmässigen Tuberkulinbehandlung Ansichten, die von den meinigen so fundamental verschieden sind und eine Verständigung so erschweren, dass ich nun notgedrungen auf dioselben noch eingeben muss.

La n d m a 11 h hat vor allem dic sondeplare Vorstellung, cin Tuber kulin sei um so besser, je giftiger es sei. Er stützt sich dabei auf das durchaus berechtigte Postulat der Serumexperimentatoren, für die Gewinnung eines kräftigen Immunserums auch kräftigo Gif te als Antigono zu verwenden. Bevor ich aber den Wort einer solchen Tuberkulintherapie mit möglichst siftigen Tuberkulinen holenehte, möchte ich darauf hinweisen, dass das momekehrto Bestroben Bor a necks, ein nicht mit übermässigen Giftwirkungen belastetes Tuberkulin zu gewinnen, sich auf schon sehr alte Erfahrungen der Pasteurschen Schule und auf die Untersuchungen von EhrIich stützen kam, wonach auch durch wenig giftige oder selbst gamz ungiftige Bakterienprodukte, durch künstlich abgeschwächte Bakteriensifte, sowie dureh sogen. Toxoile und Toxone Immunisation resp. Antikörperproduktion hervorgerufen werden kann. Es ist dies ja nach der Ehrlich schen Lehre durch die ähnliche Struktur von 'Toxinen und Toxoiden oder, wie sich dieser Forscher ausdrückt, durch die übereinstimmenden haptophoren Gruppen der beiden Arten ron Bak- 
teriensubstanzen olne weiteres zu verstehen. Bekamt genug und von grosser praktischer Tragweite ist diese Tatsache in der experimentellen Immunitätsforschung. Ehrlich hat gezeigt, dass die für das Tetanusgift so enorm empfindlichen Mäuse und Meerschweinchen nur ausserordentlich schwer mit unabgeschwächtem Tetanusgift immunisiert werden können, während ein durch Schwefelkohlenstoff abgeschwächtes Tetanusgift sich hierfür vorzüglich eignet. Auch bei der praktischen Herstellung von Immunsera gegen Tetanus und Diphtherie wird bekanntlich die Abschwächung des Diphtherie- und Tetanusgiftes durch Jodpräparate zur Erzeugung der Grundimmunität mit Erfolg benutzt. Warum sollen nun solche weniger giftige und dennoch immunisierend wirkende substanzen gerade bei der Tuberkulose nicht entweder natürlich vorkommen oder künstlich aus dem genuinen Gift hergestellt werden können und warum sollen die etwas von der grossen Heerstrasso abgehenden Versuche Boranecks, solche Substanzen aus Tuberkulosekulturen zu gewinnell, nicht gerechtfertigt sein? Warum soll ferner, da doch B e ran e ck die Möglichkeit der Herstellung solcher Extrakte experimentell bewiesen hat, denselben eine theoretische Begründung und praktische Verwendbarkeit abgosprochen werden? Rtwa bloss, weil diese Auffassung nicht in den gewohnten Gedankengang des Herrn Ja a d m a n n hineinpasst und weil ex es vorzieht, auf seiner naiven und ungemein elementaren Auffassung zu beharren, dass ein gutes Tuberkulin möglichst giftiy scin müsse, weil man ein hochwertiges I)iphtherieserum nur durch hochwertige Diphtherietoxine erzeugen kann.

Ich meinerseits stehe nicht an, umgekehrt als das Ziel der alitiven immunisatorischen Behandlung von Infektionskrankheiten geradezu die Toxoidimm unisierung hinzustellen, d. h. die Frzeugung der heilsamen immunisatorischen Reaktionen durch möglichst we u ig giftige oder ungiftige und dabei doch spezifische toxoidähnliche Substanzen, welche gleiche oder ähnliche haptophore Gruppen wie die zugehörigen Toxine, aher möglichst frei von den diese charakterisierenden toxophoren Gruppen enthalten. Freilich erscheint es mir nicht gerade wahrscheinlich, dass das Ziel der Therapie der Tuberkulose auf diesem Wege in idealer Weise erreicht werden kann. Denn, wie ich schon in meiner früheren Mitteilung mit Nachdruck hervorgehoben habe, spielt bei der Tuberkulose wenigstens in den heilbaren. Fällen die toxische Komponente in dem Krankheitsbilde nicht die stark vorwiegende Rolle wie bei anderen, akut verlaufenden Infektionskrankheiten. Infolgedessen lässt sich bei der Tuberkulose ein Teil der therapeutischen Indikationen durch Giftfestigung, wie ich stets hervorhob, überhaupt nicht erfüllen. Aber 
jedenfalls entspricht der in dem Tuberkulin B era n c c $\mathrm{k}$ beschrittene Weg weit besser dem Bedürfnis des praktischen Arztes als das ,eifrige Bestreben“ Landmanns, ,die Giftigkeit seines Tuberkulols noch zu vergrössern."

Landmann will nicht die fundamentalen Unterschiede einsehen zwischen einem Serumtier und einem zu behandelnden tuberkulösen Menschen. Und doch sind diese Unterschiede gross und einleuchtend Erstens sind die Krankhciten, gegen welche man wirksame Sera gewinnen kann, Diphtherie und Tetanus, fast reine Toxinkrankheiten, die Tuberkulose dagegen nicht. Zweitens ist das Scrumtier mehr oder weniger ein Corpus vile, was übrigens den Serumfabrikanten nicht lindert, im eigenen Interessc auch ihm anfänglich bloss abgeschwächte Gifte angedeihen zu lasson. I)rittens verfügt das Serumtier über normale Resistenzkräfte, der kranko Mensch dagegen nicht. Viertens wollen wir ja das Serumtier nicht wie den kranken Menschen heilen, sondern wir wollen zu gemeinnützigen Zwecken in seinem Blute Schutzkörper in exzessiver und greradezu unphysiologischer Menge anhäufen, während die Aufgabe der Tuberkulintherapie die jst, den Bestand des Organismus an Antikörpern gerade auf das notwendige gewissermassen physiologische Niveau oder etwas darüber hinaus zu erhöhen.

Infolge dieser Verschiedenheiten der Aufgabe kann sich der Serumfabrikant mit allfälligen anaphylaktischen oder Überempfindlichkeitserscheinungen, die sich im Verlaufe der Immunisierung bei seinen Serumtieren einstellen, abfinden, obschon er sie auch nicht gerne sieht, weil er dadurch Gefahr läuft, (reld und Arbeit zu verlieren. In der Tuberkulintherapie dagegen bedeutet das Eintreten von Giftüberlastung oder Anaphylaxie schweres Unheil und unersetzlichen Schaden an wertvollen Menschenleben. Dies ist ja bei den älteren Methoden der Tuberkulinbehandlung zwar, ,eine alte Geschichte, doch bleibt sie ewig neu, und wom sio just passieret, dem bricht sie das Herz entzwei". Diese linterschiede zwischen einem Serumpferd und einem kranken Menschen scheint La n d man n nicht einzusehen oder er berücksichtigt sie wenigstens nicht in seinen Argumenten.

Nachdem ich nun auseinandergesetzt habc, inwiefern ich das Tuberkulin Berane $\mathrm{ck}$ als thenretiseh gut begründet betrachte und auch meine Auffassungr von dem ganzen Wesen der Tuberkulinbehandlung als einer immunisatorischen Heilwirkung und nicht einer eigentlichen Immunisierung, wie sie La $n d \mathrm{~m}$ a $n$ n versteht, charakterisiert habe, bleibt es mir noch übrig, auf einige spezielle Angriffe La $\mathrm{ndmanns}$ in betreff der ron mir empfohlenen Technik der 
Tuberkulinbehandlung zu erwidern. Diese Angriffe beruhen fast alle wieder auf der fundamentalen irrtïmlichen Ansicht Landman ns von dem Zweck der Tuberkulinbehandlung.

So betrachtet es Landmann als einen ,immunisatorischen Kunstfehler", wenn ich empfehle, bei dem Übergangr von einer Lösung zur nächstfolgenden stärkeren mit der absoluten Höhe der Dose bedeutend zurückzugehen. Ich habe in meiner Arbeit erklärt, warum ich dies tue, nämlich erstens, um den die Wirkung verstärkenden Finfluss der höheren Konzentration der nenen Lösung zu kompensieren. und zweitens, um dem Organismus überhaupt immer wieder etras Ruhe zu gönnen. Beide Gründe anerkennt La n d m a n n nicht. Fr glaubt, die Konzentration, eine Giftlösung auf das Doppelte, habe bei gleicher absoluter Dosierung keinen ,messbaren Einfluss". Ich grebe zu: Dieser Einfluss ist nicht mess bar und anch nicht berechenbar. Gerade dies aber ist bedenklich, denn wem er auch nicht messbar ist, so existiert dieser Einfluss deshalb nichtsdestoweniger. Das wissen wir von chemisch definicrbaren Giften zu Genüge, wir wissen as aber auch speziell von dem Tuberkulin. Wir wissen auch genau, woher dieser Einfluss der Konzentration kommt. Der rerstorbene $\mathrm{Stochvis} \mathrm{in} \mathrm{Amsterdam} \mathrm{war} \mathrm{wohl} \mathrm{einer} \mathrm{der} \mathrm{ersten,}$ welcher dio Kalärmo dieser, für die pharmakologisehe P'osologie fundamentalen Frscheinung gab. Die Erklärung liegt einfach darin, dass konzentrierte Lösungen viel rascher in wirksamen Mengen resorbiert werden als verdünnte Lösungen. Wenn man cinwenden wollte, dass gerade für das Tuberkulin als ein langsam wirkendes (ijft, welches eine Inkubationszeit besitzt, dies nicht zutreffe, weil es für eine Giftwirkung, welche erst nach 7, 12 oder mehr Stunden klinisch manifest werde, offenbar wenig ausmachen könne, ob das Gift in einer Viertelstunde oder in einer halben Stunde resorbiert werde, so ist dies eben eine rein spekulative Überlegung, die ganz plausibcl wäre, wenn sic durch die Erfahrung bestätigt würde, die aber jeden Wert einbüsst, wenn ihr die Macht der Taisachen gegenübersteht. Fnd dies ist der Fall. Ich habo wiederholt, bevor ich meine Methode ausgearbeitet hatte, die Erfahrung machen können, dass beim Übergang von einer Lösung zur folgenden stärkeren bei gleichbleibender absoluten Dosierung die Wirkung der konzentrierteren Iösung weit heftiger war und zwar, wohlverstanden, in Fällen, wo das gleiche Bezugsdatum der beiden Lösungen bewies, dass sie aus der nämlichen Stammlösung hergestellt waren und dass also nicht etwa die stärkere Lösung auch einem stärkeren Stammgift entsprach. Warum sollte auch gerade bei Bakteriengiften die Konzentration der Lüsungen keinen Einfluss haben, während man von Morphium, Alkohol. usw. die enormen und 
eben ganz unberechenbar verstärkenden Wirkungen konzentrierterer Lösungen infolge der rascheren Resorption zur Genüge kennt? Was nun den anderen erwähnten Grund, weshalb ich jeweils beim Übergang zur stärkeren Lösung mit der absoluten Dosenhöhe erheblich zurückgehe betrifft, so erkennt ihn Herr Landmann ebenfalls nicht an. Er kann mit meiner ihm wahrscheinlich zu unwissenschaftlich klingenden Begründung, dass man dem Organismus immer wieder einige Erholung gömnen müsse, nichts anfangen und er meint, man köme eventuell eine solche Erholung leichter und billiger durch Einschaltung einer grösseren lnjektionspauso erreichen. Der Grund, warum ich solche längere Pausen nicht einschalte, wemn ich nicht durch Reaktionen dazu genötigt werde, ausser bei den grösseren Inscn, von denen man annehmen kann, dass sie länger wirken, liegt darin, dass die ganze Tendenz meiner Tuberkulinmethodo sich darin zusammenfassen lässt, dass ich mittelst derselben den natïrlichen Verlauf heilender Fälle möglichst nachahmen will. Viele klinische Beobachtungen, die sich namentlich auf die periodiseh verlaufenden Infektionskrankheiten, Pneumonie, Erysipel und Malaria beziehen, lassen sich schlechterdings nicht ohne die Anmahme erklären, dass sich negative und positive Phasen der Immunität oder wie man es jetat vielfach nennt, Immunität mol Anaphylaxie, also Giftwirkmug und Antikörperbildung bei der spontanen Feilung von Infelitionen in. periodischen Wellenlinien folgen. Tind so möchte ich auch in der Tuberkulinbehandlung diesen Wellengang des Auf- und Niedergehens der Giftwirkung nachahmen. Wenn ich nun die hierdurch nahegelegte periodische Verminderung der Dosenhöhe auf den. Moment des Überganges von einer Lösung zur nächstfolgenden stärkeren verlege, so erreiche ich dabei auch den anderen Zweck, die Wirkung der Konzentrationssteigerung, welche beim Wechsel der Lösungen vorkommt, auszuglejehen. Thd dass auch dies wünschenswert ist, habe ich soeben gezeigt. Es ist meine Ubberzeugung, dass das ganze Leben des Menschen sich in Wellenlinion rollzicht und auch auf wellenförmige Beeinflussungen eingerichtet ist, aber ich mïsste zu weit ausholen, um diese Auffassung auch Herrn La ndmann muncgerecht zu machen. Wenn er daher in diesen wohlübrlegten Eigentümlichkeiten meiner Methode einen ,immunisatorischen Kunstfehler" sieht, so kann ich meinerseits nicht umhin, in seinem mangelnden Verständnis für solche feinere Verhältnisso und in seiner fortwährenden. Verwechslung des kranken Menschen mit einem 1)iphtherieserumpferde einen Mangel an ärztlicher Einsicht zu erkemnen.

Wenn mir Landman $\mathrm{n}$ auf $\mathrm{S}$. 22 seiner Arbeit vorwirft, dass ich mit meinen eigenen Dosierungsprinzipien in Widerspruch ge- 
rate, wenn ich mir bei dem Übergang von einer Lösung zur nächst stärkeren Steigerungen von 1 auf $1^{1 / 2}$ Teilstriche, also um die Hälfte der Gesamtdose gestatte, während ich doch solche Steigerungen vorher an einer anderen Stelle ausdrücklich als zu stark bezeichnet habe, so reisst hier Lan d ma $n$ n meine Angahen aus den Zusammenliang, demn es darf nicht vergessen werden, dass eine solche Dosensteigerung um die Hälfte bei meinem Dosenschema nur vorkommt bei den ersten minimalsten Dosen, bei welchen eine Wirkung überhaupt noch gar nicht zu erwarten ist und dann ausserdem eben beim Wechsel der Lösungen und dass in dem letzteren Falle die Dosen, von welchen man ausgeht, gegenüber den vorausgegangenen erheblich reduziert worden sind, so dass dann diese etwas rasche Steigerung nur die Bedeutung einer Rekapitulation auf einer vorher in weit langsamerem 'Tempo zurückgelegten Dosierungsstrecke bedeutet. I)ie Frfalırung' lehrt, dass unter diesen Verhältnis sen solche Steigerumgen unschädlich und im Interesse des Vorwärtskommens mit der Therapie erforderlich sind und dass sie dann gerade dem von Landmann selbst mit Recht betonten und von mir keineswegs bestrittenen Prinzip der Notwendigkeit einer immunisatorischen ,Stosswirkung", d. h. einer Stimulation der natürlichen Heilfakturen, entsprechen. Dieser wirkliche Sachverhalt wird durch die von $L$ a $n d m$ a $n$ n aufgestellte Dosentabelle vollständig verdunkelt, indem durch die unübersichtliche Form derselben und weil die Angaben der Lösungskonzentrationen bloss in Anmerkungen zur Tabelle mitgeteilt werden, der Unkundige durch diese Tabelle zu ganz irrtümlichen Vorstellungen von meiner Dosierung verleitet wird. Die Landmann schen Tabellen wirken überhaupt gegenüber meiner ganz klaren Darstellung höchst verwirrend. Warum bezeichnet z. B. Landmann die D eny ssche Dosierungsskala in der Rubrik B seiner Tabelle als ,S Sh lis gewöhnliche Art"? Warum zieht er ferner, um das Gelände noch mehr zu verdunkeln, die Bandelier sche Skala heran, ron der ich in meiner Arbeit kein Wort gesagt habe? Mit welchem Recht ferner stellt er meiner Skala eine von ihm selbst nach einem ganz anderen Prinzip, nämlich nicht nach einfachen Volumsverhältnissen der verwendeten Lösungen, sondern nach absoluten Giftmengen konstruierte und die Konzentrationen nicht berücksichtigende, deshalb gar nicht vergleichbare Skala gegenüber, eine Skala, die sich nicht, wie ich es als praktisch erforderlich hingestellt habe, mechanisch nach genau ablesbaren Spritzenteilstrichen und einfachen Bruchteilen derselben durchführen lässt, sondern die Berücksichtigung der zweiten Dezimale bedarf, die niemand in einer Injektionsspritze messen kann, eine Skala also, die ir der Hand des Praktikers notwendigerweise Irrtümer herbei- 
Noch ein Wort über die von mir für die Beurteilung einer Tuberkulinbehandlung als wichtig hervorgehobene Bedeutung der Hautreaktionen an der Injektionsstelle. Jeder erfahrene Tuberkulintherapeut, speziell jeder, der das B r a ne $\mathrm{cksche}$ Tuberkulin verwendet hat, weiss, dass in der Tat die Hautreaktionen für die Beurteilung des Immunitätszustandes sehr wichtig sind und diese Auffassung ist ja durch die Erfahrungen über die Pirquetsche Methode der endematischen Tuberkulindiagnostik bestätigt worden. Landmann tut diese Erfahrungen mit der Bemerkung ab, das Wesen dieser Hautreaktionen sei noch unbekannt und müsse weiter studiert werden, was ich natürlich durch ihre praktische Verwertung durchaus nicht verhindern möchte, und er benutzt die Gelegenheit, um auch hier mich in höchst unmotivierter Weise anzugreifen, indem er meint, die von mir beobachteten Hautreaktionen seien auf ungenügende Antisepsis zurückzuführen. Er gibt mir bei diesem Anlass Räte, in welcher Weise das ihm offenbar schwierig erscheinende Problem, subkutane Injektionen aseptisch und antiseptisch durchzuführen, gelöst werden könne. Fr stösst sich nämlich daran, dass ich in der deutschen Ausgabe meiner Arbeit empfahl, die - wohlverstanden natürlich vorher ausgekochte - Spritze in 3\% iger Borax 10 sungauf $z u b e w a h r e n$ und dass ich die Haut mit Sublimatlösung bloss bet u p f e, statt sie „kräftig abzureiben“. Diese meine Angaben kommen La n d m a n n so ungeheuerlich vor, dass er nicht umhin kann, sie durch zweiAusrufungszeichen zu brandmarken. Ich bemerke dazu, dass die Aufbewahrung steriler Instrumente in $3 \%$ iger Boraxlösung nicht von mir erfunden ist, sondern von namhaften Chirurgen als ein die Asepsis schon sterilisierter Instrumente gewährleistendes und die Instrumente konservierendes Verfahren empfohlen wurde. Ich habe allerdings seit ungefähr zwei Jahren den Borax aufgegeben, nicht weil seine Wirkung als die Sterilität konservierendes (nicht etwa sterilisierendes) Mittel mit ungenügend schien, sondern weil ich durch Herrn Beraneck aufmerksam gemacht wurde, dass die dem Spritzeninnern anhaftende Boraxlösung infolge ihrer leicht alkalischen Beschaffenheit bei kleinen Injektionsdosen durch Neutralisation der sauren Tuberkulinlösung leicht Fällungen hervorrufen kann. In der neueren französischen Ausgabe ist die Boraxlösung deshalb definitiv durch Alkohol ersetzt, in welchem die sterile Spritze aufbewahrt wird, was allerdings den Nachteil hat, dass jedesmal vor dem Gebrauch die Spritze durch steriles Wasser vom Alkohol befreit werden muss, da auch Alkohol das Tuberkulin Beraneck fällt. Ich habe übrigens auch schon in der deutschen Auflage dieses Alkoholverfahren gegenüber dem Boraxverfahren als noch sicherer und ansserdem, da wo sich die Sache 
durchführen lässt, als das Beste auch das je desmalig e Auskochen der Spritze empfohlen. Ich muss es also ablehnen, dass meine Hautreaktionen mit fehlerhafter Spritzenasepsis etwas zu tun haben. Auch die Bemängelung des „Betupfens" der Haut mit Sublimatlösung ist durchaus nicht gerechtfertigt. Ich pfege dieses auf meiner Klinik auch bei allen Punktionen angewandte und bewährte Verfahren den Studenten dadurch zu begründen, dass ich ihnen erkläre, dass eine sichere Desinfektion der Haut bekanntlich überhaupt ein noch ungelöstes Problem ist, dass es ferner bei so kleinen Eingriffen auf eine solche Desinfektion gar nicht ankommt, sondern dass der Zweck eines Antiseptikums bei Punktionen nur der sein kann, in die Stichwunde hinein eine Spur einer antiseptischen, d. h. hier entwickelungshemmenden Substanz zu bringen, welche das Hineinwuchern von Bakterien verhindern soll. Zu diesem Zweck wird bei allen unseren Punktionen durch eine Schicht sublimatlösung hindurchgestochen und nach der Entfernung der Kanüle ein Tropfen sublimatlösung whe jegliches Reiben auf die Stichwunde, getupft". Wir bedienen uns dabei einer alkoholischen Sublimatlösung, trotzdem sich eine solche zur eigentlichen Desinfektion bekanntlich schlechter eignet, als eine wässrige. Denn wir wollen ja aber hier gar nicht desinfizieren, somdern wir wollen einfach Sublimat nach der Verdunstung des Alkohols in minimaler Menge in Substanz in der Stichwunde deponieren. Hierzu eignet sich gerade die alkoholische Lösung, weil bekanntlich nur eine solche sicher auf der fettigen Haut haftet. Fin kräftiges Abreiben der Haut mit einem Antiseptikum vor der Punktion würde höchstens die Wirkung haben, dass man nachher beim Einstechen Eipidermisschuppen, die doch nicht sicher sterilisiert sind, mittelst der Kanüle in die Stichwunde hineinschleppt und ich pflege vor diesem Verfahren meine Studenten geradezu zu warnen. Zum mindesten ist das Reiben, für welches sich Herr $\mathrm{I}_{\text {a }} \mathrm{n} \mathrm{dm}$ m n n so begeistert, völlig zwecklos. Es wäre also wünschenswert, wenn Herr La $n$ dmann bei seinen Kranken auch so verfahren würde wie ich, statt mir Lehren über I'unktionsintisepsis zu geben.

Der von Herrn La an dman $n$ gegen das Prinzip, die gebrauchsfertigen Lösungen zu beziehen, erhobene Vorwurf, dass solche verdünnte Lösungen nicht haltbar seien, ist natürlich hinfällig, da jede Lösung von dem Laboratorium B e r a n e $\mathrm{k}$ wegen des Fehlens eines antiseptischen Zusatzes immer erst auf die erfolgte Bestellung hin ad hoc unmittelbar vor der Versendung hergestellt wird. Wie lange diese Lösungen damn haltbar sind, habe ich in meiner Arbeit angegeben. Dass ron einem Lagern der Lösungen in Depots und bei Zwischenhändlern nicht die Rede ist, ergibt sich daraus, dass solche 
überhaupt in Anbetracht des Umstandes, dass die Lösungen nur frisch zur Verwendung kommen, nicht existieren und dass die Lösungen nur direkt vom Herstellungsort bezogen werden können.

Endlich noch ein Wort über die Vergleichung der maximalen Giftdosen, welche bei den verschiedenen Verfahren der Tuberkulinbehandlung nach der Berechnung Landmanns dem kranken Menschen im Verlauf der Tuberkulinbehandlung einverleibt werden. Landman $\mathrm{n}$ kommt zu dem Resultat, dass in Form seines Tuberkulols und bei seiner Methode der Dosierung der Patient 5000 mal mehr Gift erhalte, als nach der von mir empfohlenen Methode mit dem Beraneckschen Tuberkulin. Abgesehen nun davon, dass Landman n für das Tuberkulin Beraneck gar nicht über die nötigen Daten verfügt, um eine solche Vergleichung einwandsfrei vorzunehmen, da die letale Giftdosis des Tuberkulin Beraneck am gesunden Meerschweinchen, wie ich früher hervorhob, bisher wegen der geringen Giftigkeit dieses Präparates und der aus diesem Grunde erforderlichen grossen Menge der injizierten Flüssigkeit sich nicht bestimmen liess, muss ich den Wert einer solchen Vergleichung prinzipiell durchaus ablehmen. Ich betone eben nochmals, dass ich die Tuberkulinbehandlung nicht rom Standpunkte einer einfachen Immunisierung aus betrachte, sondem vom Standpunkte der von mir sogenannten immunisatorischen Heilwirkung. Denn wenn ich auch die Ursache der günstigen Tuberkulinwirkung in Giftfestigung sehe, so ist damit noch lange nicht gesagt, dass nun die therapeutische Wirkung unter allen Umständen um so grösser sei, je höher die Giftfestigung ist. Diese braucht bloss g e $n \ddot{u} g$ end zu sein. Es ist dies namentlich einleuchtend, wenn man bedenkt, dass die Giftfestigung stets nur durch vorübergehende negative Phasen der Immunität, mit anderen Worten durch Schädigungen erworben werden kann. Mit diesen, für die Tuberkulinbehandlung fundamentalen, sie von der gewöhnlichen Immunisierung trennenden Gedanken sollte man sich endlich einmal vertraut machen, sonst kommt man immer wieder auf Abwege und läuft Gefahr, seine Kranken dauernd zu schädigen. Auch bei der Tuberkulinbehandlung wie bei jeder anderen überlegt und geschickt durchgeführten Therapie gilt der Satz: Kleine Ursachen, grosse Wirkungen. Heroische Mittel dagegen schiessen oft weit über das Ziel hinaus. Wenn also L a n d man $n$ darauf pocht, dass er seinen Patienten im Verlauf seines Behandlungsplanes, auf tödliche Meerschweinchendosen berechnet, eine 5000 mal grössere Giftmenge angedeihen lässt als ich, so ist dies nach dem Gesagten an sich noch gar kein Lob seiner Therapie und kein Tadel der meinigen. Wenn $L$ a $n \mathrm{~d} m$ a $n \mathrm{n}$ dies dennoch meint, so lässt er dabei ausserdem auch völlig das für die Therapie im Gegensatz zu den

Beiträge zur Klinik der Tuberkulose. Bd. X. H. 4. 
Verhältnissen des Serumpferdes so wichtige und oben von mir betonte Prinzip der Toxoidimmunisierung ausser acht. Ich kann mich infolgedessen auch nicht der sehr kühnen Folgerung von Lan d m a n n (S. 24 seiner Arbeit) anschliessen, dass sein Tuberkulin $5000 \mathrm{mal}$ mehr leiste als das Tuberkulin B e r a n e ck, weil das dabei verabfolgte Tuberkulin einen $5000 \mathrm{mal}$ grösseren Giftwert repräsentiere. Für diese nicht gerade durch Bescheidenheit sich auszeichnende Behauptung ist Landmann den Beweis völlig schuldig geblieben und sie steht gewiss nicht recht im Verhältnis zu demjenigen, was überhaupt sich mit der Tuberkulinbehandlung erzielen lässt. Denn wenn man die Erfolge des Tuberkulin Beraneck auch noch so bescheiden bemisst, so müsste, falls wirklich die Erfolge des Tuberkulol Landmann 5000 mal besser wären, die Tuberkulose sich durch dieses Mittel mit der grössten Leichtigkeit aus der Welt schaffen lassen und in dem Wirkungskreis des Herrn Landmann wäre sie wohl seit seinen Mitteilungen in den Jahren 1898 und 1900 schon erloschen.

In diesem Zusammenhang darf ich nun wohl nochmals auf das ,wissenschaftliche Mäntelchen" zurückkommen, von dem ich in meiner Tuberkulinarbeit sagte, dass es gewissen Anwendungsformen der Tuberkulins umgehängt werde, wenn man nach absoluten Giftwerten, die auf das gesunde Meerschweinchen berechnet sind, dosiert. Herr Landmann hat mir diese Bemerkung, wie es scheint, sehr übel genommen. Ich glaube nicht ganz mit Recht. Offenbar nahm Herr La $\mathrm{n} d \mathrm{~m}$ a $\mathrm{n} \mathrm{n}$ an, ich bezeichne die Wertbestimmung der Tuberkuline an gesunden Tieren als ein wissenschaftliches Mäntelchen. Aus dem ganzen Zusammenhang und auch aus dem Wortlaut meiner Darstellung geht aber vielmehr hervor, dass ich den Nutzen einer solchen Wertbestimmung vollkommen anerkenne, sonst hätte ich eine solche nicht, wie ich dort anführte, für das schweizerische Tuberculinum Kochi veranlasst und für das Tuberkulin Beraneck wenigstens, wenn auch aus den angeführten Gründen ohne Erfolg, versuchen lassen. Freilich halte ich eine solche Wertbestimmung nur für nützlich in dem Sinne, dass man dadurch die Wahrscheinlichkeit einer konstanten Zusammensetzung des betreffenden Tuberkulins erhöht. Das war also mit dem wissenschaftlichen Mäntelchen nicht gemeint. Gemeint war vielmehr, wie ich es auch ausdrücklich sagte, das Rekordrennen nach hohen, in Meerschweinchentoxineinheiten ausgedrückten Tuberkulindosen. Den Vorwurf, so zu verfahren, habe ich nun damals Landman n keineswegs gemacht, sondern ich hatte bloss ganz allgemein die Gefahr im Auge, dass, wie es für alles in der Medizin Modeströmungen gibt, so es auch Mode werden könnte, den Leuten damit imponieren zu wollen, dass man ihnen vorrechnet, wie viele tödliche Meer- 
schweinchendosen an Tuberkulin sie schon ertragen. Dass eine solche Besorgnis, die sich übrigens bei mir auf gewisse Erfahrungen stützt, bei den heutigen medizinischen Strömungen nicht ganz unberechtigt ist, wird mir gewiss der Leser zugeben. Der Vorwurf war also ein ganz unpersönlicher und dabei antizipierter, gewissermassen prophylaktischer. Wenn also damals das wissenschaftliche Mäntelchen Herrn Lan dman n völlig kalt lassen konnte, weil e r es ja nicht angezogen hatte, so betrübt es mich jetzt um so mehr, zu hören, dass er Miene zu machen scheint, sich damit zu drapieren, wenn er als besonderen Vorzug rühmend hervorhebt, dass er bei seiner Tuberkulolbehandlung den Kranken einen 5000 mal höheren Giftwert inkerporiert als ich mit meinem Verfahren.

Mit der nach meiner Ansicht irrtümlichen Ansicht La n d m a n n s von der Tuberkulintherapie als einer einfachen Immunisation stehen die Einwände im Zusammenhang, welche er gegen das von mir aufgestellte individuelle Maximum und Optimum der Tuberkulindosierung macht. Diese Einwände erledigen sich für denjenigen, welcher mich wirklich verstanden hat und verstehen will, von selbst. Auch der Vorwurf Landmanns, dass ich in manchen Beziehungen unbestimmt in der Formulierung meines Heilplanes sei, beruht auf der Verkennung des ärztlichen Prinzips der individualisierenden Behandlung und ich akzeptiere ihn gern, nicht als einen Vorwurf allerdings, sondern als ein Lob. Die Therapie lässt sich überhaupt nicht in Schablonen zwingen. Sie erfordert die ganze geistige Tätigkeit des Arztes für jeden einzelnen Fall. Darum erzielt der eine mehr, der andere wenig therapeutische Erfolge mit den nämlichen Mitteln. Der Meissel allein macht nicht die Statue, sondern der Künstler muss ihn auch zu handhaben wissen.

Ich glaube, damit die wesentlicheren Einwendungen Landmanns widerlegt zu haben. Auf alle Entstellungen, welche der La n d m a n n sche Artikel sonst noch enthält, mag ich nicht eingehen. Meine Erwiderung ist im Verhältnis zum Gewicht der Lan dmannschen Argumente schon ohnehin viel zu lang geworden. Für jeden aufmerksamen Leser meiner Arbeit erledigt sich der Rest von selbst. Namentlich habe ich keinen Grund, auf Dinge einzugehen, welche mit dem Streitobjekt nichts zu tun haben und welche La n d m a n n offenbar nach dem Grundsatz ,semper aliquid haeret", „der Zweck heiligt die Mittel", herbeizieht, um mir am Zeuge zu flicken. Dahin gehört sein Angriff auf die, wie ich erwähnte, von mir als Mitglied der eidgenössischen Pharmakopoekommission veranlasste, aber keineswegs von mir ausgearbeitete Wertbestimmung des schweizerischen K o ch schen Tuberkulins. Die Methode dieser Prüfung ist überhaupt 
gar nicht publiziert worden und es ist mir deshalb auch ganz unverständlich, wie Landmann, ohne sie zu kennen, dazu kommt, sie zu kritisieren und somit einen Windmühlenkampf gegen mich aufzuführen. Er mag die Verantwortung dieser Prüfung ruhig den wissenschaftlichen Leitern des schweizerischen Seruminstituts überlassen, die sicherlich in diesen Fragen kompetent genug sind. Dieser ganze Passus zeigt, wie wenig wählerisch Landmann in der Wahl seiner Kampfmittel ist.

Auch auf alle möglichen Prioritätsreklamationen des Herrn La $\mathrm{n} d \operatorname{man} \mathrm{n}$ anderen und mir gegenüber will ich nicht näher eingehen. Meine Arbeit hatte nur den Zweck, aufklärend zu wirken und ich habe für mich keinerlei Prioritätsansprüche erhoben. Ich lehnte es auch in meiner Arbeit ausdrücklich ab, eine vollständige Übersicht über die Geschichte der Tuberkulinbehandlung zu geben. Wenn aber Herr Landmann gegenüber Götsch in betreff der vorsichtigen Dosierung des Tuberkulins für sich selbst Prioritätsansprüche erhebt, so muss ich hierzu doch bemerken, dass dies mit den in seinen Arbeiten mitgeteilten, seine Dosierung betreffenden Zahlen kaum in Einklang zu bringen ist. Im übrigen erwähne ich im Interesse der historischen Gerechtigkeit, dass hier keinem anderen als Lichtheim die eigentliche Priorität zukommt, wie in vielen anderen Fragen, in welchen die Literatur bahnbrechende Ideen dieses Autors totgeschwiegen hat. Lichtheim war, wie ich selbst leider erst nach dem Erscheinen meiner Arbeit erfuhr, der allererste Autor, welcher die reaktionslose Tuberkulinbehandlung empfahl und zwar schon gleich nach dem Bekanntwerden des Tuberkulins ${ }^{1}$ ). Ich

1) Klin. Jahrbuch 1891, Ergänzungsband, „Die Wirksamkeit des Koch schen Heilmittels“ S. 634 und Deutsche med. Wochenschr. 1891.

Lichtheim sagt an der erstzitierten Stelle wörtlich folgendes: „Sehr bald haben wir, wie auch viele andere Beobachter, die Erfahrung gemacht, dass bei schweren wie bei leichten Phthisen bei allmählicher Steigerung der Dosen die Reaktionserscheinungen völlig ausbleiben können. Als nun markante Zeichen der Besserung ausblieben, kam uns das Bedenken, ob dieses Verfahren das richtige sej, ob nicht die Reaktion, der Entzündungssturm die unerlässliche Bedingung der Rückbildung sei. Eine Prüfung dieser Frage an Lupuskranken hat uns gezeigt, dass diese bedenken ungerechtfertigt waren. Wir haben zwei Lupusfälle in derselben Weise behandelt. In dem einen traten schon bei geringfügigen Dosen Fiebererscheinungen auf, doch blieb die lokale Reaktion eine sehr mässige, in dem anderen fehlte die allgemeine Reaktion vollkommen, die lokale war sehr geringfügig und beschränkte sich auf eine leichte Rötung der Ränder der erkrankten Partien. In beiden Fällen traten sehr manifeste Rückbildungsprozesse frühzeitig auf.

Auf Grund dieser Beobachtungen halte ich es für zweckmässig, die Reaktionserscheinungen bei Phthisikern, soweit dies möglich ist, vollkommen zu vermeiden." 
habe diese mir früher entgangene Tatsache in der französischen Ausgabe meiner Tuberkulinarbeit nachgetragen.

Dies meine Erwiderung. Das fadenscheinige Gewebe der La n dmannschen Gründe wird für den kundigen Leser nicht verstärkt durch 'den ausgiebigen Gebrauch von Kraftausdrücken, die er sich gestattet, wie ,völlig verkehrt", „wertlos", „,öllig missglückt", „heillose Verwirrung", „überschwängliches Lob des Tuberkulin Beraneck", ,bedauerlicher Rückschritt der Tuberkulinbehandlung", usw. Der Leser wird den Wert einer derartigen Polemik ohne weiteren Kommentar zu würdigen wissen. 